\title{
Begriffe und Kategorien vergleichender Forschung. Einige kritische Bemerkungen
}

\author{
Csaba Makó
}

1. Sozialpolitisch und theoretisch orientierte Leitgedanken in der vergleichenden Arbeit

\subsection{Sozialpolitische Implikationen vergleichender Forschung}

Seit dem Ende der 70er Jahre, noch stärker aber in den 80er Jahren, läßt sich bei ungarischen Industriesoziologen ein wachsendes Interesse an den verschiedenen Formen gesellschaftlicher und betrieblicher Krisenanpassung beobachten. Hinter diesem Interesse steht die recht allgemeine Erkenntnis, daß die gegenwärtige Industriekrise das Verhältnis der Sozialpartner zueinander, d.h. die Struktur der Beziehungen zwischen Arbeitgebern, Arbeitnehmern und dem Staat, grundlegend verändert. Diese Beziehungsmuster wandeln sich auf nationaler, regionaler und lokaler Ebene. Die nationalen Reaktionen auf die Krise bzw. die *Formen der Krisenanpassung* provozierten eine Vielfalt von Kooperationsformen und von höchst unterschiedlichen Managementstrategien. Die Entwicklung der industriellen Beziehungen in den letzten 2 Jahrzehnten hat die Vorstellung eines sogenannten *one best way * für die wirtschaftliche und soziale Umstrukturierung ad absurdum geführt.

Das bedeutet, daß gegenüber Patentrezepten, die tendenziell jeweils einen Aspekt der Krisenanpassung überbetonen, Vorsicht geboten ist. Hinsichtlich der flexiblen Nutzung von Arbeitskraft heißt dies etwa, daß selbst die nachstehende Folgerung der OECD nicht schlicht akzeptiert werden kann:

„... für die Flexibilisierung des Arbeitsmarktes gibt es kein spezifisches Maßnahmen-Paket, das für alle Länder gleichermaßen geeignet wäre ... es gibt mehrere Wege, zu den Zielen Zielen effektive Anpassung, beständige Innovation, höhere Beschäftigung und bessere Lebensqualität.* (Dahrendorf 1986, S. 9)

Die andere Seite der Flexibilität, insbesondere Arbeitsplatzverlust, Arbeitslosigkeit, Arbeitsintensivierung (Verschärfung der Leistungsanforderungen), wechselnde Beschäftigungspolitik usw. sind im obigen Statement der OECD *unterbelichtet火.

Theorieorientierte vergleichende Forschung zu sozialpolitischen Problemen kann helfen, die spezifischen Verknüpfungen von sozial-ökonomischen und kulturell-ideologischen Regelungen der Krisenbewältigung in den beteiligten Länder besser zu verstehen. So hat z.B. eine vergleichende Untersuchung klei- 
nerer und mittlerer Betriebe in Süditalien nicht nur die allgemein bekannten Interdependenzen unterschiedlicher Regulierungsmechanismen aufgezeigt, sondern auch deren lokale Besonderheiten und deren wechselnde Bedeutung über einen längeren Zeitraum hinweg: *Ohne spezielle Vereinbarungen zur sozialen und politischen Kooperation > vor Ort > kann die Politik wirtschaftlicher Umstrukturierung durch die Sozialpartner nicht erfolgreich umgesetzt werden.* (Simonyi 1988, S. 1)

\subsection{Einige methodologische Schwierigkeiten vergleichender Forschung}

In theoretischer Sicht ist international vergleichende Forschung durch eine $»$ doppelte Herausforderung* gekennzeichnet: Erstens: Entscheidende Bedeutung hat zunächst eine intensive theoretische und methodologische Vorbereitung. Die *Workshop-Diskussion* ist ein Weg, um einen akzeptablen Konsens über das Projektziel und über die Interpretation der zentralen Untersuchungsvariablen zu erreichen. Massive Konsensdefizite torpedieren die vergleichende Datenanalyse: Selbst im Falle gut vorbereiteter und hochmotivierter nationaler Forscherteams führt die Forschung dann lediglich zu einem Nebeneinander von mehr oder weniger gut angefertigten nationalen Monographien - wie dies etwa im Falle des wohlbekannten *Automations- und Industriearbeiter*-Projektes des Vienna Center (Grootings 1986) geschehen ist.

Zweitens: Zentrale Zielsetzung auch vergleichender Forschung ist die Validierung des benutzten Ansatzes (bzw. des »Paradigma*). Eine systematisch-kritische Diskussion der verschiedenen nationalen Perspektiven wollte helfen wider Wahrnehmungsverengung und *neue Orthodoxie* (z.B. bezüglich des Zusammenhanges von , flexibler Spezialisierung • und neuen Qualifizierungspolitiken. Vergleichende Forschung erfordert - anders ausgedrückt - die kontinuierliche kritische Auseinandersetzung um theoretisch-analytische Konzepte und Methodologien. Vergleichende Forschungsarbeit zum Technologietransfer hat etwa zur Zurückweisung sowohl des *technizistischen* wie des *kulturalistischen* Interpretationsansatzes geführt.

Untersuchungen zeigen, daß die gleiche Technologie deutlich verschieden genutzt wird, je nachdem, ob es sich um ein diese Technologie exportierendes oder importierendes Land handelt. Der makro-ökonomische Kontext und die sozio-kulturellen und historischen Institutionen prägen entscheidend die gesellschaftlichen Produktionsstrukturen und die Modi der Arbeitsteilung. Unterstrichen wird durch diese und andere Sachverhalte als zentrale Anforderung an vergleichende Forschung: Eine kontinuierliche Überarbeitung der Erhebungsinstrumente als Merkmal dieses neuen Typus von industriesoziologischer Forschung. 


\section{Einige Beispiele zur Veranschaulichung der Schwierigkeiten eines Ver- gleichs}

Bei der Durchführung vergleichender Studien sind Industriesoziologen häufig mit folgenden Problemen konfrontiert: Ein und dieselbe Berufsbezeichnung hat je nach national-historischem Kontext des untersuchten Betriebes eine andere Bedeutung. Kritisch kann dies bei der Untersuchung betrieblicher Arbeitskraftnutzungs- und Aufstiegspolitik werden. Mit Blick etwa auf die in der Elektronik-Industrie durchgeführten Denki Roren-Erhebungen zeigt sich, daß in japanischen Betrieben viele technische Angestellte (Techniker und Ingenieure) vergleichsweise hochrangige *logistische* Verantwortung zu übernehmen haben, während demgegenüber in den ungarischen und polnischen Betrieben ein nur sehr geringer Anteil der technischen Angestellten (1,7\%-3,2\%) Aufgaben wie Produktionsplanung und Produktionsvorbereitung übernehmen. (Mako/Maurice 1987). Ein anderes international vergleichendes Projekt - das M.I.T. Auto Industry Project - hat ganz ähnliche Sturkturmuster des Arbeitskräfteeinsatzes aufgezeigt. So war etwa in den deutschen Automobilwerken die Zahl der *Verfahrensingenieure $=$ rund doppelt so hoch wie die der *Produktionsingenieure* (vgl. hierzu auch Jürgens, in diesem Band).

Für ein angemessenes Verständnis der geltenden sozialen Regulierungsmechanismus ist es schließlich auch wichtig, den unterschiedlichen allgemeinen sozialen Grundlagen des *Arbeitslebens* in den einzelnen Ländern stärkere Beachtung zu schenken als dies üblich ist; Es ist z.B. häufig dies landesspezifisch vorherrschende Struktur des Familienverbandes, die Sippe , ein bedeutender im Hinblick auf die Wahl der Faktor für Berufs- und Arbeitsplatzwahl:

*Aktuelle, wie historische Beispiele belegen die Bedeutung, die der Familie bei der Arbeitskräfterekrutierung in der Industrie zukommt. * (Whipp-Grieco 1983, S. 14)

\begin{tabular}{|c|c|c|c|}
\hline  & $\begin{array}{l}\text { Die Verteilung de } \\
\text { Wirtschaftssektore } \\
\text { (Arbeitsstunden in }\end{array}$ & $\begin{array}{l}\text { beitszeit } \\
\text { liarden) }\end{array}$ & ch \\
\hline Sektoren/Jahre & 1967 & 1980 & 1984 \\
\hline Transport & 8,2 & 7,9 & 7,8 \\
\hline Haushalt & 3,0 & 2,9 & 2,9 \\
\hline Second Economy & $3,3 \quad 4,0$ & 4,3 & 4,6 \\
\hline , First Economy ،* & 9,9 & 9,8 & 9,1 \\
\hline Gesamt & $25,0 \quad 25,1$ & 24,9 & 24,4 \\
\hline
\end{tabular}

* „Socialist Sector* - Bereich der sozialistischen Planwirtschaft

Quelle: Timàr, J. (1985, S. 1636). 
Damit nicht genug der Komplikation: Die wachsende Bedeutung etwa der sog. "second economy* in Ungarn, erfordert, daß diese in künftigen vergleichenden Forschungsvorhaben berücksichtigt wird. Die vorstehende Tabelle veranschaulicht die wachsende Bedeutung der *second economy* und der sich in ihr herausbildenden lokal- und national-spezifischen Regulierungsformen.

\section{Schlußbemerkungen}

Immer wieder richten Industriesoziologen ihr Interesse auf die technische und organisatorische Entwicklungsdynamik des Arbeitsprozesses. Jüngst wird dies beispielsweise durch die Aktualität des Topos *flexible Spezialisierung* verdeutlicht (Piore/Sabel 1985; Kern/Schumann 1984). Angesichts der sozialen Dynamik in den verschiedenen Wirtschaftssektoren - nicht nur in der Industrie, sondern auch im Dienstleistungsbereich - gilt es wohl für den Zweck vergleichender Studien die tradierte mextensive Forschungsweise* in der Industriesoziologie, die vor allem mit standardisiserten Forschungstechniken arbeitet, zu ergänzen. Erstens erhalten systematische Expertengespräche zu den technischen und organisatorischen Veränderungen mit betrieblichen 'Schlüsselfiguren' und mit den Vertretern der Sozialpartner zentralen Stellenwert im Forschungsdesign. Zweitens ist ein intensiver Arbeitszusammenghang zwischen den an der vergleichenden Forschung beteiligten Wissenschaftlern unerläßlich. Es ist dies nicht nur eine Frage von regelmäßigen Kontakttreffen, sondern es muß so etwas wie ein *Forschungsteam als Prozeß* etabliert werden. Schließlich gelingt es nur bei Kombination von verschiedenen Forschungs-Ansätzen und bei Nutzung unterschiedlicher Forschungsinstrumente, der großen Gefahr rein quantitativ verfahrender empirischer Forschung zu entgehen: *Immer mehr und mehr über immer weniger und weniger $\mathrm{zu}$ wissen.* 